\title{
Use of Monitoring Indicators in Hospital Management of Antimicrobials
}

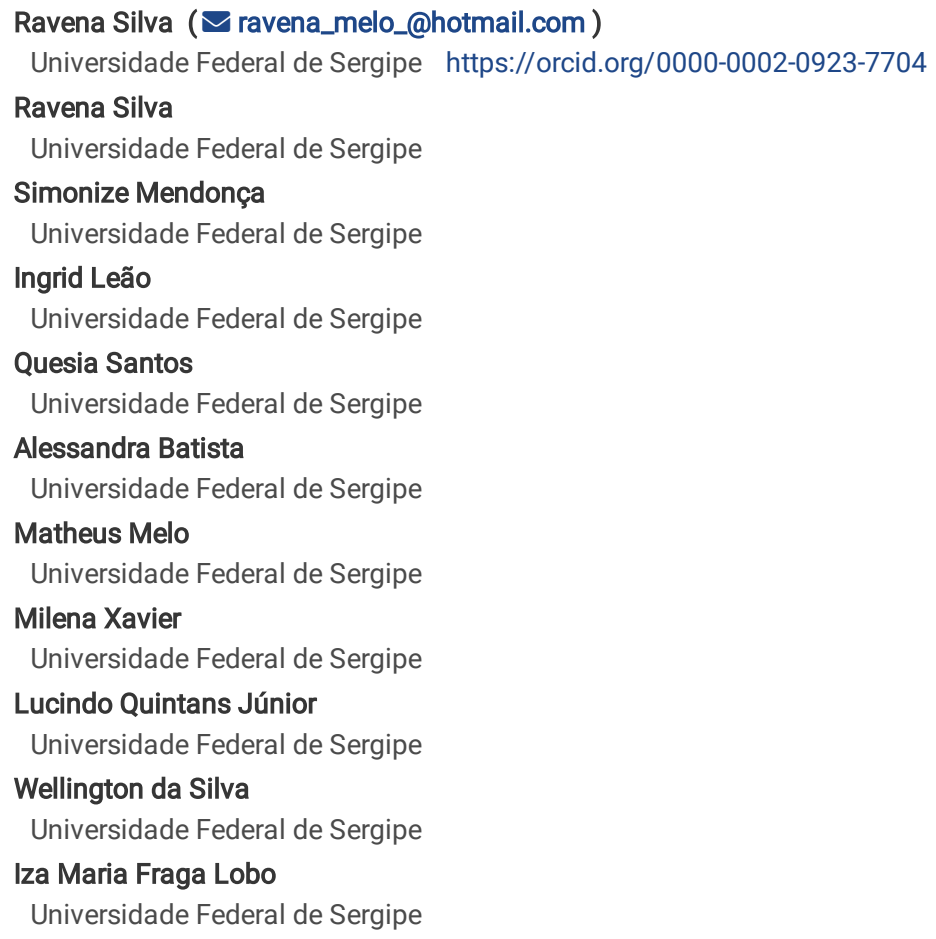

Version of Record: A version of this preprint was published at BMC Infectious Diseases on August 17th, 2021. See the published version at https://doi.org/10.1186/s12879-021-06542-5. 


\section{Abstract}

Background: It is described in the literature that between $25 \%$ to $50 \%$ of antimicrobial prescriptions, in the hospital environment, are unnecessary or inadequate, directly impacting antimicrobial resistance. The National Health Surveillance Agency launched the "Brazil Stewardship Project" to assess the national panorama of antimicrobial use management programs, therefore, it is expected that managers will be encouraged to implement the antimicrobial use management program, with the publication of the results, enabling the comparison of national data. In this context, measures and indicators of antimicrobial use will be particularly important. We demonstrate the application of the indicators DOT, LOT e DOT/LOT ratio to explore and monitor antimicrobials use.

Methods: This is an observational, analytical and prospective study conducted in a teaching hospital, with 94 active beds, distributed between the intensive care unit (ICU), the surgical clinic (SUR), the medical clinic (MED), the pneumology/infectology (PNE/INF) and pediatrics (PED).

Results: During the study period, a total of 11,634 patient-days were followed up and an average of $50.4 \%$ of patients were exposed to some antimicrobial, showing significant reduction throughout the year. In 376 days, for every 1,000 days of hospitalization, patients were receiving antimicrobial therapy (LOT = $376 / 1000 \mathrm{pd}$ ). Overall, the $1 \mathrm{st}$ generation cephalosporins and fluoroquinolones were highlighted both in the number of prescriptions and in the duration of therapy. The calculated global DOT / LOT ratio showed that each patient received an average of 1.5 antimicrobials during the hospital stay. The incidence of antimicrobial resistance, globally, for both Methicillin R and Carbapenem R, was 1 per 1000 patient-days.

Conclusions: The indicators addressed in this study proved to be of great relevance for obtaining data regarding the use of antimicrobials, contributing to the initiatives to be taken by the Stewardship program. This study is expected to encourage other hospitals to monitor the use of antimicrobials, allowing comparison of data.

\section{Background}

One of the main public health problems worldwide is microbial resistance, a process that, although it is a natural biological phenomenon, is occurring at an accelerated rate due to the excessive use of antimicrobials (ATMs). It is described in the literature that between $25 \%$ to $50 \%$ of antimicrobial prescriptions, in the hospital environment, are unnecessary or inadequate, increasing hospital stay, morbidity, health care costs, and directly impacting antimicrobial resistance [1-6].

The absence of guidelines on the rational use of antimicrobials, uncertainty about the differential diagnosis, incorrect choice of medication, improper treatment duration, presence of complex comorbidities, incorrect interpretation of microbial results and lack of surveillance to monitor the use of antimicrobials are factors that imply the inappropriate use of these drugs $[5,7,8]$.

Because of the above, the Centers for Disease Control and the World Health Organization, together with the United Nations member countries, including Brazil, have been encouraging the implementation of programs such as the "Antimicrobial Stewardship Program", which is a set of interventions, audits, and feedback, to optimize the use of ATMs [2,3,9,10]. Recently, in July 2019, the National Health Surveillance Agency launched the "Brazil Stewardship Project" [6], to assess the national panorama of antimicrobial use management programs, encouraging managers to implement antimicrobial use management programs, with the publication of the results, enabling the comparison of national data.

Several measures have been used to evaluate ATM use and consumption. The Defined Daily Dose (DDD), which expresses the daily dose, in grams, of a drug used for its main indication in adults is the oldest and most widely used, however, this measure is not recommended for pediatric populations, as individual doses vary according to the child's weight. Thus, alternative metrics were proposed and adopted, such as "Days of Therapy" (DOT), equivalent to the number of days a patient receives an antimicrobial agent (regardless of the dose) and "Length of therapy " (LOT), referring to the number of days the patient is on antimicrobial therapy (regardless of the number of drugs). The higher the DOT and LOT the longer the patient is exposed to the use of antimicrobials. In contrast, the DOT / LOT ratio, this can be useful to assess the number of antimicrobials prescribed per patient $[1,3,9,11,12]$.

In this context, measures and indicators of antimicrobial use will be particularly important. We demonstrate the application of the indicators DOT, LOT e DOT/LOT ratio to explore and monitor antimicrobials use. Brazil still has scarce information on the use of antimicrobials at the patient level and, to date, this is the first study at the national level that uses the DOT and LOT indicators compiling several hospital wards. Therefore, the objective of the present study is to map the use of antimicrobials, in a university hospital in northeastern Brazil, using DOT and LOT, to stimulate further research with the application of these indicators.

\section{Methods}

\section{Design and setting}

This is an observational, analytical and prospective study conducted in a teaching hospital, with 94 active beds, distributed between the Intensive Care Unit (ICU), the Surgical Clinic (SUR), the Medical Clinic (MED), the Pneumology/Infectology (PNE/INF) and Pediatrics (PED).

As of the 2 nd quarter of the study, the hospital started implementing actions in the new Stewardship program, which were carried out gradually and progressively throughout the year. The program involved a joint and integrated work of professionals from the Health Care-Related Infection Control Service, Care Risk Management Unit, Hospital Pharmacy, Microbiology, Infectology, Intensive Care, Pediatrics, and others specialties, in a joint effort to implement and monitor measures to improve the use of antimicrobials. 
The prescription for groups of drugs called "audited" (amikacin, ampicillin + sulbactam, azithromycin, cefepime, cefotaxime, ceftazidime, ceftriaxone, ciprofloxacin, clindamycin, fluconazole, levofloxacin, metronidazole, moxifloxacin, teicoplanin, and vancomycin) and "restricted" (ertapenem, imipenem+cilastatin, meropenem, and micafungin) it involved the assessment and previous audit of the management team, authorizing or not the use and indicating the duration of the therapy.

\section{Data Source}

A monthly analysis of the prescriptions of a sample of adult and pediatric patients admitted to the wards (including those who did not receive antimicrobials) was carried out between 1 January and 31 December 2018. The review of prescriptions and data extraction was performed by the researcher, using an electronic database created especially for this purpose. The data regarding the use of antimicrobials were obtained from the individual prescriptions of the patients and forms of requests for antimicrobials, under the responsibility of the hospital pharmacy.

\section{Inclusion and exclusion criteria}

Data analysis included antimicrobial agents administered intravenously or orally. The exclusion criteria involved patients with a stay of fewer than 24 hours, use of intramuscular, topical, ophthalmologic, inhaled, antiviral, and antiretroviral antimicrobials. Preoperative antimicrobial prophylaxis given in the operating room under protocol does not appear in the ordinary prescription and, therefore, was excluded from the analysis.

\section{Outcome measures}

Primary outcomes were represented by the percentage of patients using antimicrobials, and the number of antimicrobial prescriptions by class. Secondary outcomes included analyzes of antimicrobial use, individually and by class, expressed in DOT, and LOT per 1000 patient-days (pd), the DOT / LOT ratio, and the incidence of Methicillin-Resistant Staphylococcus aureus (Methicillin ${ }^{\mathrm{R}}$ ) and Carbapenem Resistance in Klebsiella pneumoniae, Pseudomonas aeruginosa, and Acinetobacter baumannii (Carbapenem ${ }^{\mathrm{R}}$ ) per 1000 patient-days $(\mathrm{pd})$, and diagnostics.

\section{Statistical analysis}

Data entry and storage were performed in Microsoft Excel 2019 (Microsoft Corporation, Redmond, WA, USA), transferred for statistical analysis using Stata software version 15.1 (StataCorp, College Station, Texas, USA). The variables were expressed as means and percentages. The normality of data distribution was verified and compared using the ANOVA test or the Kruskal-Wallis test with respective post-tests, as appropriate.

For the analysis of time trend, generalized linear regression was used by the Prais-Winsten model, which allows the correction of first-order serial autocorrelation, indicated for time series analysis. The level of statistical significance was set at 0.05 .

\section{Results}

During the study period, a total of 11634 patient-days was followed up and an average of $50.4 \%$ of patients was exposed to some antimicrobial (Additional file 1). The intensive care unit was the ward that contained the largest proportion of patients using ATM (86\%; ICU vs SUR, MED, PNE / INF, PED; $p$ <0.05), followed by pneumology/infectology (62\%; PNE / INF vs SUR, MED; $p<0.05)$ and pediatrics (61\%; PED vs SUR, MED; $p<0.05)($ Table 1$)$.

Overall, the most prescribed classes were the 1st generation cephalosporins (25.0\%), fluoroquinolone (13.2\%), and carbapenems (8.4\%) (Additional file 1). The 1st generation cephalosporins were the most prescribed classes both in surgical clinic (49.9\%; SUR vs MED, PNE / INF, ICU; $p<0.05)$ and in pediatrics (34.5\%; PED vs MED, PNE / INF; p <0.05). The medical clinic had a higher number of prescriptions referring to 3rd generation cephalosporins (14.7\%; MED vs SUR, ICU; $p$ <0.05), pneumology/infectology had a higher number of antifungals (16\%; PNE / INF vs SUR, PED; $p<0.05)$ and the ICU prevailed the prescription of carbapenems (19.2\%; ICU vs SUR, MED, PNE / INF, PED; $p<0.05)$ (Table 1).

In 376 days for every 1000 days of hospitalization, patients were receiving antimicrobial therapy (LOT = 376/1000pd) (Additional file 1). Comparing hospital wards, the ICU was where patients were exposed to ATMs for the longest time (753 DOT / 1000pd; ICU vs SUR, MED, PED; p <0.05), followed by pneumology/infectology (470 DOT / 1000pd; PNE / INF vs SUR, MED; p <0.05). The calculated global DOT / LOT ratio showed that each patient received an average of 1.5 antimicrobials during the hospital stay (Additional file 1), with a higher number within the ICU (2 DOT / LOT; ICU vs SUR, MED, PNE / INF, PED; $p$ $<0.05)$ (Table 1).

Table 1. Distribution of use antimicrobial, by hospital ward, 2018. 


\begin{tabular}{|c|c|c|c|c|c|c|}
\hline \multirow[t]{2}{*}{ VARIABLES } & \multicolumn{6}{|c|}{ WARDS } \\
\hline & SUR & MED & PNE/INF & PED & ICU & p-value \\
\hline Use of antimicrobials (\%) & $38^{a}$ & $42^{\mathrm{b}}$ & $62^{c(a b)}$ & $61^{\mathrm{d}(\mathrm{ab})}$ & $86^{\mathrm{e}(\mathrm{abcd})}$ & $0.000 *$ \\
\hline \multicolumn{7}{|c|}{ Number of prescriptions (\%) } \\
\hline First-generation cephalosporins IV/PO & $49.4^{\mathrm{a}(\mathrm{bce})}$ & $3.8^{\mathrm{b}}$ & $1.4^{\mathrm{c}}$ & $34.5^{\mathrm{d}(\mathrm{bc})}$ & $9.4^{\mathrm{e}}$ & $0.000^{*}$ \\
\hline Fluoroquinolones IV/PO & $20.5^{\mathrm{a}}$ & $14.1^{\mathrm{b}}$ & $11.2^{\mathrm{c}}$ & $3.6^{\mathrm{d}(\mathrm{abe})}$ & $13.5^{\mathrm{e}}$ & $0.000^{*}$ \\
\hline Carbapenems IV & $1.7^{\mathrm{a}(\mathrm{bc})}$ & $11.5^{\mathrm{b}}$ & $8.8^{\mathrm{c}}$ & $6.3^{d}$ & $19.2^{\mathrm{e}(\mathrm{abcd})}$ & $0.000 *$ \\
\hline Third-generation cephalosporins IV & $6.5^{\mathrm{a}}$ & $14.7^{\mathrm{b}(\mathrm{ae})}$ & $8.5^{\mathrm{C}}$ & $9.2^{\mathrm{d}}$ & $4^{\mathrm{e}}$ & $0.004^{*}$ \\
\hline Metronidazole IV/PO & $10.1^{\mathrm{a}}$ & $5.8^{\mathrm{b}}$ & $1.7^{\mathrm{c}(\mathrm{ae})}$ & $6.3^{d}$ & $11.1^{\mathrm{e}}$ & $0.031^{*}$ \\
\hline Cefepime IV & $0.8^{\mathrm{a}}$ & $10.3^{\mathrm{b}(\mathrm{a})}$ & $15.6^{\mathrm{c}(\mathrm{ade})}$ & $6.0^{d}$ & $7.1^{\mathrm{e}}$ & $0.000 *$ \\
\hline Clindamycin IV & 9.1 & 7.1 & 5.1 & 2.1 & 7.4 & 0.072 \\
\hline Antifungals IV/PO & $0.2^{\mathrm{a}}$ & $7.1^{b(a)}$ & $16^{\mathrm{c}(\mathrm{ad})}$ & $4.5^{d}$ & $6.7^{\mathrm{e}}$ & $0.000^{*}$ \\
\hline Glycopeptides IV & $1.1^{\mathrm{a}}$ & $6.4^{b}$ & $3.4^{\mathrm{c}}$ & $3^{d}$ & $10.4^{\mathrm{e}(\mathrm{acd})}$ & $0.001 *$ \\
\hline Azithromycin IV/PO & $0^{\mathrm{a}}$ & $2.6^{b}$ & $9.5^{\mathrm{c}(\mathrm{abe})}$ & $6.3^{\mathrm{d}(\mathrm{ae})}$ & $1.7^{\mathrm{e}}$ & $0.000 *$ \\
\hline Penicillins ${ }^{1}$ IV/PO & $0.2^{\mathrm{a}}$ & $10.3^{\mathrm{b}(\mathrm{ae})}$ & $3.4^{\mathrm{c}}$ & $8.3^{\mathrm{d}(\mathrm{ae})}$ & $1^{e}$ & $0.000 *$ \\
\hline Co-trimoxazole IV/PO & $0^{\mathrm{a}}$ & $3.2^{\mathrm{b}}$ & $10.9^{c(a b e)}$ & $3.6^{d(a)}$ & $3^{e}$ & $0.000 *$ \\
\hline Aminoglycosides IV & $0.4^{\mathrm{a}}$ & $2.6^{b}$ & $4.4^{\mathrm{C}}$ & $6.5^{d(a)}$ & $3.4^{\mathrm{e}}$ & $0.025^{\star}$ \\
\hline LOT/1000pd & $300^{a}$ & $262^{b}$ & $470^{c(a b)}$ & $382^{d}$ & $753^{e(a b d)}$ & $0.000 *$ \\
\hline \multicolumn{7}{|l|}{ DOT/1000pd } \\
\hline Fluoroquinolones IV/PO & $108^{a}$ & $57^{b}$ & $71^{c}$ & $24^{d}$ & $147^{e(b d)}$ & $0.001^{*}$ \\
\hline First-generation cephalosporins IV/PO & $123^{a(b c)}$ & $5^{b}$ & $6^{c}$ & $94^{\mathrm{d}(\mathrm{bc})}$ & $73^{\mathrm{e}(\mathrm{bc})}$ & $0.000 *$ \\
\hline Carbapenems IV & $11^{\mathrm{a}}$ & $43^{b}$ & $84^{\mathrm{c}(\mathrm{a})}$ & $40^{\mathrm{d}}$ & $368^{e(a b d)}$ & $0.000^{*}$ \\
\hline Third-generation cephalosporins IV & 43 & 50 & 64 & 61 & 35 & 0.775 \\
\hline Cefepime IV & $8^{a(c e)}$ & $32^{\mathrm{b}}$ & $95^{c}$ & $47^{d}$ & $114^{\mathrm{e}}$ & $0.000 *$ \\
\hline Metronidazole IV/PO & $56^{\mathrm{a}}$ & $12^{\mathrm{b}}$ & $8^{c(a d)}$ & $59^{d}$ & $119^{\mathrm{e}(\mathrm{bc})}$ & $0.000 *$ \\
\hline Antifungals IV/PO & $1^{\mathrm{a}(\mathrm{ce})}$ & $24^{\mathrm{b}}$ & $83^{c}$ & $40^{d}$ & $118^{\mathrm{e}}$ & $0.000 *$ \\
\hline Co-trimoxazole IV/PO & $0^{a(\text { cde })}$ & $4^{b(c)}$ & $121^{\mathrm{c}}$ & $25^{d}$ & $72^{\mathrm{e}}$ & $0.000 *$ \\
\hline Clindamycin IV & 47 & 28 & 37 & 12 & 75 & 0.099 \\
\hline Glycopeptides IV & $8^{a}$ & $25^{\mathrm{b}}$ & $36^{c}$ & $10^{d}$ & $183^{e(a b c d)}$ & $0.000 *$ \\
\hline Aminoglycosides IV & 6 & 16 & 32 & 34 & 83 & 0.055 \\
\hline Penicillins ${ }^{1}$ IV/PO & $1^{a(b d)}$ & $35^{b}$ & $18^{c}$ & $38^{d}$ & $13^{e(b d)}$ & $0.000^{*}$ \\
\hline Azithromycin IV/PO & $0^{a(c d)}$ & $8^{b(c)}$ & $40^{c}$ & $31^{d}$ & $23^{e}$ & $0.000^{*}$ \\
\hline DOT/LOT ratio & $1.4^{\mathrm{a}}$ & $1.3^{b}$ & $1.5^{\mathrm{c}}$ & $1.4^{\mathrm{d}}$ & $2.0^{e(a b c d)}$ & $0.000 *$ \\
\hline
\end{tabular}

Subtitle: SUR - Surgical Clinic, MED - Medical Clinic, PNE/INF - Pneumology / Infectology, PED - Pediatrics, ICU - Intensive Care Unit. 1 penicillin/penicillin with beta-lactamase inhibitor.

$\mathrm{a}, \mathrm{b}, \mathrm{c}, \mathrm{d}, \mathrm{e}-$ statistical differences per line (Bonfferroni $\left.{ }^{*} \mathrm{p}<0.05\right)$

In the hospital, the therapy time was longer for fluoroquinolone (75 DOT / 1000pd), followed by 1st generation cephalosporins (66 DOT / 1000 pd), and carbapenems (61 DOT / 1000pd) (Additional file 1). Exposure time to 1st generation cephalosporins in the surgical clinic (123 DOT / 1000pd; SUR vs MED, PNE / INF; p <0.05) and in pediatrics (94 DOT / 1000pd; PED vs MED, PNE / INF; p <0.05) was superior to the other antimicrobial classes. Higher exposures were also observed for fluoroquinolone in the medical clinic (57 DOT / 1000pd), co-trimoxazole in pneumology/infectology (121 DOT / 1000pd) and carbapenems in the ICU (368 DOT / 1000pd; ICU vs SUR, MED, PED; $p<0.05)$ (Table 1).

The incidence of antimicrobial resistance, globally, for both Methicillin ${ }^{R}$ and Carbapenem ${ }^{R}$, was 1 per 1000 patient-days (Additional file 1 ), presenting higher rate of Carbapenem ${ }^{R}$ in ICU (3; ICU vs SUR, PNE / INF, PED; $\left.p<0.05\right)$ (Table 2).

Table 2. Incidence of antimicrobial resistance per 1000 patient-days, by hospital ward, 2018.

\begin{tabular}{lcccccc}
\hline ANTIMICROBIAL RESISTANCE & \multicolumn{6}{c}{ WARDS } \\
\cline { 2 - 7 } & SUR & MED & PNE/INF & PED & ICU & p-value \\
\hline Methicillin $^{\mathrm{R}}$ & 0 & 1 & 2 & 0 & 3 & 0.101 \\
Carbapenem $^{\mathrm{R}}$ & $0^{\mathrm{a}}$ & $2^{\mathrm{b}}$ & $0^{\mathrm{c}}$ & $0^{\mathrm{d}}$ & $3^{\mathrm{e}(\mathrm{a}, \mathrm{c}, \mathrm{d})}$ & $0.005^{\star}$ \\
\hline
\end{tabular}

Subtitle: SUR - Surgical Clinic, MED - Medical Clinic, PNE/INF - Pneumology / Infectology, Methicillin ${ }^{\mathrm{R}}$ - Methicillin-resistant Staphylococcus aureus Carbapenem ${ }^{R}$ - Carbapenem Resistance in Klebsiella pneumoniae, Pseudomonas aeruginosa, and Acinetobacter baumannii $\mathrm{a}, \mathrm{b}, \mathrm{c}, \mathrm{d}, \mathrm{e}$ - statistical differences per line (Bonfferroni *p <0.05)

Overall, diagnoses for the use of antimicrobials involved surgical prophylaxis (35.6\%), respiratory infections (23.6\%), and sepsis (13.9\%) (Additional file 1). Surgical prophylaxis was the main indication for the use of antimicrobials in the surgical clinic ( $80.8 \%$; SUR vs MED, PNE / INF; $p<0.05)$ and in pediatrics (36.2\%; PED vs MED, PNE / INF; p <0.05). Respiratory infections were higher in the medical clinic (22.4\%) and pneumology/infectology (39.1\%; PNE / INF vs 
SUR; $p<0.05)$, while in the ICU the diagnosis of sepsis prevailed $(34.1 \%$; ICU vs SUR; $p<0.05)$. There was no significant difference in the diagnosis of skin / soft tissue infections, urinary infections, abdominal infections, and others (Table 3).

Table 3. Distribution of diagnostic for the use of antimicrobials, by hospital ward, 2018.

\begin{tabular}{lcccccc}
\hline \multirow{2}{*}{$\begin{array}{c}\text { DIAGNOSTIC } \\
\text { (\%) }\end{array}$} & \multicolumn{7}{c}{ WARDS } \\
\cline { 2 - 7 } & SUR & MED & PNE/INF & PED & ICU & p-value \\
\hline Surgical prophylactic & $80.8^{\mathrm{a}(\mathrm{bc})}$ & $0^{\mathrm{b}}$ & $0^{\mathrm{c}}$ & $36.2^{\mathrm{d}(\mathrm{bc})}$ & $33.9^{\mathrm{e}}$ & $0.000^{*}$ \\
\hline Respiratory infection & $4.2^{\mathrm{a}}$ & $22.4^{\mathrm{b}}$ & $39.1^{\mathrm{c}(\mathrm{a})}$ & $32.9^{\mathrm{d}}$ & $19.8^{\mathrm{e}}$ & $0.017^{*}$ \\
\hline Sepsis & $0^{\mathrm{a}}$ & $19.0^{\mathrm{b}}$ & $19.6^{\mathrm{c}}$ & $5.4^{\mathrm{d}}$ & $34.1^{\mathrm{e}(\mathrm{a})}$ & $0.033^{*}$ \\
\hline Skin/soft tissue infection & 2.5 & 17.2 & 16.3 & 10.1 & 0.9 & 0.154 \\
\hline Urinary infection & 2.5 & 19.0 & 14.1 & 5.4 & 3.8 & 0.749 \\
\hline Abdominal infection & 7.5 & 19.0 & 1.1 & 0.7 & 7.5 & 0.134 \\
\hline Others & 2.5 & 3.4 & 9.8 & 9.4 & 0 & 0.141 \\
\hline
\end{tabular}

Subtitle: SUR - Surgical Clinic, MED - Medical Clinic, PNE/INF - Pneumology / Infectology, PED - Pediatrics, ICU - Intensive Care Unit. Others - Surgical Site Infection, Visceral Leishmaniasis, Febrile Neutropenia, Bacterial Endocarditis.

$\mathrm{a}, \mathrm{b}, \mathrm{c}, \mathrm{d}, \mathrm{e}-$ statistical differences per line (Bonfferroni ${ }^{*} \mathrm{p}<0.05$ )

Trend

The time series analysis reveals a significant reduction in the use of antimicrobials, globally $(-1.0 \% ; p<0.05)$, and in particular, in the surgical clinic $(-1.6 \% ; p<0.05)$, on the other hand, there was an increase in the medical clinic $(2.6 \% ; p<0.05)$ (Additional file 1 ; Additional file 2). Overall, there was a significant decrease in the use of aminoglycosides $(-0.3 \% ; p<0.05)$, and a significant increase for carbapenems $(0.4 \% ; p<0.05)$. The surgical clinic obtained a significant reduction in the number of aminoglycosides prescriptions $(-0.1 \% ; p<0.05)$. In the medical clinic, there was a reduction in the number of penicillins $(-1.9 \% ; p<0.05)$ and an increase in the number of carbapenems $(1.0 \% ; p<0.05)$. In pneumology / infectology, reductions were observed for 1 st generation cephalosporins $(-0.3 \% ; p<0.05)$ and aminoglycosides $(-1.1 \% ; p<0.05)$. In pediatrics, there was an increase in the number of azithromycin prescriptions $(0.9 \%$; $p$ $<0.05$ ). There was no significant change in the ICU prescriptions (Additional file 1; Additional file 2).

There were no significant changes in the duration of antimicrobial therapy (LOT/1000pd) and the DOT / LOT ratio, in general, and between the units analyzed (Additional file 1; Additional file 2). Regarding the time of use single of antimicrobials, in hospital, significant decreases were observed for use of 1st generation cephalosporins (-2.9 DOT/1000pd; $p<0.05)$, and aminoglycosides $(-0.4$ DOT/1000pd; $p<0.05)$. These falls were mostly observed in the surgical clinic (1st generation cephalosporins -10.6 DOT/1000pd and aminoglycosides -1.8 DOT/1000pd; p <0.05), with increased time for use of glycopeptides (1.7 DOT/1000pd; $p<0.05)$. In the medical clinic, there was reduced the time in the use of penicillins $(-4.9 \mathrm{DOT} / 1000 \mathrm{pd} ; \mathrm{p}<0.05)$ and extended the therapy to carbapenems (5.6 DOT/1000pd; $p$ <0.05). In pneumology / infectology, the only significant change observed was a reduction in the time of use of aminoglycosides (-7.3 DOT/1000pd; $p<0.05)$. In pediatrics, azithromycin use time increased $(3.6 \mathrm{DOT} / 1000 \mathrm{pd} ; \mathrm{p}<0.05)$. In the ICU there was a decrease in the time of use of clindamycin (-23.5 DOT/1000pd; $p<0.05)$, and an increase for carbapenems $(24.4$ DOT/1000pd; $p<0.05)$.

Overall, incidence rates of antimicrobial resistance revealed an important increase of Methicillin ${ }^{R}(0.1 ; p<0.05)$, especially in the surgical clinic $(0.1 ; p$ $<0.05)$ and in the ICU (1.0; $\mathrm{p}<0.05)$ (Additional file 1; Additional file 2).

\section{Discussion}

The present study found that an average of $50.4 \%$ of patients was exposed to some antimicrobial. A large study carried out by Versporten [7], when evaluating the use of antimicrobials in patients admitted to adult hospitals in 53 countries, identified, in Latin America, the average use of $36.8 \%$, ranging from $32.5 \%$ to $43.4 \%$. However, the literature describes a broader range that extends from $22 \%$ to $76 \%$ of hospitalized patients receiving at least one antimicrobial during hospital stay $[1,4,8,9,12]$. Even though the result, observed here, is within the presented range, these studies demonstrate variations that may be related to the type of care provided in each hospital, where the need for antimicrobial indication may be higher or lower.

The greatest number of patients exposed to the use of antimicrobials was in the intensive care unit ( $86 \%)$, followed by pneumology/infectology ( $62 \%)$ and pediatrics $(61 \%)$. On the other hand, time trend analyses showed a reduction in the percentage of use of antimicrobials in the surgical clinic and an increase in the medical clinic. The predominance of the use of ATMs in the ICU was also observed in a study of one-off prevalence on health-related infections in Austria, in which $67.9 \%$ of patients in this ward were exposed to some antimicrobial, other studies indicate variations from $55.1 \%$ to $57 \%[1,4,7]$. Variations are also observed in the medical clinic (31.8\% - 73.2\%), and in the surgical clinic $(37.3 \%$ to $84.1 \%)[1,7,8,13]$. The fragile conditions with which patients arrive at the ICUs explain the greater frequency of infections and, consequently, the greater need for antibiotic therapy in this ward.

In 376 days, for every 1000 days of hospitalization, patients were receiving antimicrobial therapy (LOT $=376 / 1000$ pd), with the longest time observed in the ICU (753 DOT / 1000pd), followed by pneumology/infectology (470 DOT / 1000pd). The LOT indicator is rarely found in the literature to express the use of antimicrobials, however, a study in the United States of America found an average LOT of 536 (median, 529; range, 427-684) per 1000 patient-days in 70 American hospitals [12], a value above that observed in this study. In addition to the gap in publications on the different monitoring measures for antimicrobial 
management programs, the lack of disclosure of monitoring strategies and measures in hospitals in low and middle-income countries stands out. A recent meta-analysis of 221 studies using clinical trials, interrupted time series, and other methods evaluated the effectiveness of antibiotic management programs, in which unfortunately few studies represent these countries [14].

The ICU and pneumology/infectology presented LOTs well above the overall hospital rate, reflecting the severity and frequency of community and hospital infections in patients assisted in these two wards, which require more frequent, intense, and longer antimicrobial therapy. The calculated global DOT / LOT ratio showed that each patient received an average of 1.5 antimicrobials during the hospital stay, with a higher number prescribed within the ICU (2 DOT / LOT). The literature points to similar values ranging from 1.3 to 2.1 antimicrobials per patient $[4,5,8]$.

Overall, the 1st generation cephalosporins and fluoroquinolones were highlighted both in the number of prescriptions and in the duration of therapy. The 1st generation cephalosporins were the most prescribed classes and had longer use time at the surgical clinic and in pediatrics, presenting a significant reduction in the surgical clinic. The medical clinic had a higher number of prescriptions referring to third-generation cephalosporins with longer exposure to quinolones, these data showed reductions over the year, but without significant change. On the other hand, an increase important was observed in both the number of prescriptions and the time of exposure to carbapenems. In the pneumology/infectology predominated the use of antifungals and longer use for cotrimoxazole, with irrelevant increases monthly. Despite this, there was a significant reduction in the use of aminoglycosides. In the ICU, carbapenems stood out both in the number of prescriptions and the time of use, with a significant increase in this exposure throughout the study.

The use of 1 st generation cephalosporins and quinolones in the hospital under study is related to the surgical profile of the institution, with the adoption of protocols that are still old. However, the data reveal that there is a reductive trend in the use of these drugs. In early January 2019, European Medicines Agency released a report recommending the suspension and restriction of the use of some antibiotics, among them quinolones and fluoroquinolones, because of the serious, disabling, and potentially permanent side effects that these drugs have presented. The literature also reports a significant correlation between the use of cephalosporins and fluoroquinolones with the increase in carbapenem-resistant Enterobacteriaceae [15,16].

A study conducted by Kimura [17], which evaluated the long-term effects of antibiotic administration programs at a university hospital in Japan, found that therapy days per 1000 patient-days were higher for 1 st generation cephalosporins (45 DOT / 1000pd). The time of use of quinolones (4.7 DOT / 1000 pd) and carbapenems (13.5 DOT / 1000pd) were well below the values verified in this study. However, studies show results ranging from 25.8 DOT / 1000 pd to 132.3 DOT / 1000pd for quinolones and 8.7 DOT / 1000pd to 39.1 DOT / 1000pd for carbapenems $[2,9,10]$, this variation may be related to the lack of uniformity in data collection, which implies the importance of standardizing the obtainment of these rates, allowing reliable comparability of the data.

Another relevant data refers to the increase in the use of carbapenems in the hospital, mainly in the ICU. The high use of carbapenems in the ICU can be explained by the high incidence of Carbapenem ${ }^{R}$. Also, there is an increase of Methicillin ${ }^{R}$ in the ICU and the surgical clinic. Until then, there is no expectation about the availability of new antibiotics to replace carbapenems. The implementation and adherence to simple infection control measures such as hand hygiene, the correct cleaning of the environment, and contact precautions still work as basic strategies to prevent the transmission of multi-resistant bacteria [16].

The surgical prophylaxis, respiratory infections, and sepsis were the most described diagnoses for the use of antimicrobials. The surgical clinic and pediatrics pointed out surgical prophylaxis as the main diagnostic for the use of antimicrobials $(80.8 \%$ and $36.2 \%$, respectively), which is in line with the greater number of indications of $1 \mathrm{st}$ generation cephalosporins in these wards. Highlights for the prophylactic use of antimicrobials, as observed in this study, were reported by Talaam [5], in a rural hospital in western Kenya, where prophylaxis was responsible for $56.3 \%$ of antimicrobial indications. Gutema [8], documented that the prophylactic use of antimicrobials represented $41.3 \%$ in the prescriptions in the surgical clinic and $8.5 \%$ in the medical clinic.

Respiratory infections prevailed in the medical clinic and pneumology/infectology $(22.4 \%$ and $39.1 \%$, respectively), this data reflects the characteristics of the wards described, and corroborate with studies that describe that approximately $50 \%$ of the use of antimicrobials is used in the treatment of respiratory and urinary infections $[4,8,9,13]$. In the ICU the main diagnostic indications were for the treatment of sepsis $(34.1 \%)$. Sepsis is a serious public health problem in ICUs in Brazil, this pathology is the second leading cause of mortality within this environment. Empirical treatment with antibiotics is usually initiated with broad-spectrum drugs, such as carbapenems, observed in this study, possibly due to the number of multidrug-resistant strains isolated in these patients [18].

The strengths of are the study prospective design with the observation of time series, a direct investigation of the prescriptions that allow greater analysis of the use of antimicrobials, and the use of DOT and LOT indicators according to the new tools for monitoring the use of antimicrobials. However, some weaknesses were observed: First, the study was conducted at a single center. Second, they have not evaluated the comorbidities. Third, the adequacy of the prescriptions was not addressed.

\section{Conclusion}

The indicators addressed in this study proved to be of great relevance for obtaining data regarding the use of antimicrobials, contributing to the actions of feedback of information in the process of continuing education of health professionals, to be taken by the Stewardship program, recently implemented in the hospital, to improve patient care and safety. All data found were in accordance with the ranges observed in the literature, which implies the accuracy of the information. This study is expected to encourage other hospitals to monitor the use of antimicrobials, allowing comparison of data.

\section{Abbreviations}

ATM - Antimicrobials

Carbapenem ${ }^{\mathrm{R}}$ - Carbapenem Resistance in Klebsiella pneumoniae, Pseudomonas aeruginosa, and Acinetobacter baumannii 
DDD - Defined Daily Dose

DOT - Days of Therapy

ICU - Intensive Care Unit

LOT - Length of Therapy

MED - Medical Clinic

Methicillin ${ }^{\mathrm{R}}$ - Methicillin-resistant Staphylococcus aureus

PED - Pediatrics

PD - Patient-days

PNE/INF - Pneumology/Infectology

SUR - Surgical Clinic

\section{Declarations}

Ethics approval and consent to participate. This study was approved by the Research Ethics Committee of the Federal University of Sergipe, with CAAE: 74181317.8.0000.5546, under opinion No. 2,256.79. A waiver of informed consent was obtained due to the non-interventional nature of this study and the complete anonymity of patient data.

Consent for publication. Not applicable.

Availability of data and materials. All data generated or analyzed during this study are included in this published article [and its supplementary information files].

Competing interests. The authors declare that they have no competing interests.

Funding. Not applicable.

Authors' contributions. RMRS and SCBM collected, analyzed, and interpreted the data, and wrote the work. QNS, AMB, MSM, and INL helped with data collection. LJQJ and MMX reviewed and collaborated on the work structure. WBS and IMFL contributed to the conception of the work and substantially revised it. All authors read and approved the final manuscript.

Acknowledgments. Not applicable.

\section{References}

[1] Nathwani D, editor. Antimicrobial stewardship: from principles to practice. British Society for Antimicrobial Chemotherapy. http://www.bsac.org.uk/antimicrobialstewardshipebook/BSAC-AntimicrobialStewardship-FromPrinciplestoPractice-eBook.pdf. Published 2018.

[2] Morrill HJ, Caffrey AR, Gaitanis MM, LaPlante KL. Impact of a prospective audit and feedback antimicrobial stewardship program at a veterans affairs medical center: A six-point assessment. PLoS One. 2016; doi:10.1371/journal.pone.0150795.

[3] Agência Nacional De Vigilância Sanitária (ANVISA). Diretriz nacional para elaboração de programa de gerenciamento do uso de antimicrobianos em serviços de saúde.

http://portal.anvisa.gov.br/documents/33852/271855/Diretriz+Nacional+para+Elabora\%C3\%A7\%C3\%A3o+de+Programa+de+Gerenciamento+do+Uso+de+An 7edc-411b-a7e0-49a6448880d4. Published 2017. 
[4] Segagni LL, Blacky A, Starzengruber P, Diab-Elschahawi M, Wrba T, Presterl E. A national point prevalence study on healthcare-associated infections and antimicrobial use in Austria. Wien Klin Wochenschr. 2016; doi:10.1007/s00508-015-0947-8.

[5] Talaam RC, Abungana MM, Ooko PB. An antibiotic audit of the surgical department at a rural hospital in Western Kenya. Pan Afr Med J. 2018; doi:10.11604/pamj.2018.29.219.14510.

[6] Agência Nacional De Vigilância Sanitária (ANVISA). Projeto stewardship brasil. Avaliação nacional dos programas de gerenciamento do uso de antimicrobianos em unidade de terapia intensiva adulto dos hospitais brasileiros.

http://portal.anvisa.gov.br/documents/33852/271855/Projeto+Stewardship+Brasil/435012dc-4709-4796-ba78-a0235895d901. Published 2019.

[7] Versporten A, Zarb P, Caniaux I, Gros MF, Drapier N, Miller M, Jarlier V, Nathwani D, Goossens H. Antimicrobial consumption and resistance in adult hospital inpatients in 53 countries: results of an internet-based global point prevalence survey. Lancet Glob Heal. 2018; doi:10.1016/S2214-109X(18)30186-4.

[8] Gutema G, Håkonsen H, Engidawork E, Toverud EL. Multiple challenges of antibiotic use in a large hospital in Ethiopia - A ward-specific study showing high rates of hospital-acquired infections and ineffective prophylaxis. BMC Health Serv Res. 2018; doi:10.1186/s12913-018-3107-9.

[9] Baggs J, Fridkin SK, Pollack LA, Srinivasan A, Jernigan JA. Estimating national trends in inpatient antibiotic use among US hospitals from 2006 to 2012. JAMA Intern Med. 2016; doi:10.1001/jamainternmed.2016.5651.

[10] Dubrovskaya Y, Scipione MR, Siegfried J, Jen SP, Pham V, Papadopoulos J, Decano A, Lewis T, Dabestani A. Multilayer model of pharmacy participation in the antimicrobial stewardship program at a large academic medical center. Hosp Pharm. 2017; doi:10.1177/0018578717726993.

[11] Antimicrobial Stewardship Programs (ASPS) - Metrics Examples (Public Health Ontario).

https://www.publichealthontario.ca/en/eRepository/ASP_Metrics_Examples.pdf. Published 2017.

[12] Polk RE, Hohmann SF, Medvedev S, Ibrahim O. Benchmarking risk-adjusted adult antibacterial drug use in 70 US academic medical center hospitals. Clin Infect Dis. 2011; doi:10.1093/cid/cir672.

[13] Fortini A, Faraone A, Di Pietro M, Cappugi C, Magnante G, Boccadori C, Bartolini S, Rabatti L. Antimicrobial stewardship in an Internal Medicine ward: effects on antibiotic consumption and on the use of carbapenems. Intern Emerg Med. 2018; doi:10.1007/s11739-018-1916-9.

[14] Davey P, Marwick CA, Scott CL, Charani E, McNeil K, Brown E, Gould IM, Ramsay CR, Michie. Interventions to improve antibiotic prescribing practices for hospital inpatients. Cochrane Database Syst Rev. 2013; doi:10.1002/14651858.CD003543.pub3.

[15] European Medicines Agency (EMA). Human medicines: highlights of 2018. https://www.ema.europa.eu/en/documents/report/human-medicineshighlights-2018_en.pdf. Published 2019.

[16] European Centre for Disease Prevention and Control (ECDC). Rapid risk assessment: Carbapenem-resistant Enterobacteriaceae - first update. Stockholm: ECDC. https://ecdc.europa.eu/sites/portal/files/documents/RRA-Enterobacteriaceae-Carbapenems-European-Union-countries.pdf. Published 2018. 
[17] Kimura T, Uda A, Sakaue T, Yamashita K, Nishioka T, Nishimura S, Ebisawa K, Nagata M, Ohji G, Nakamura T, Koike C, Kusuki M, loroi T, Mukai A, Abe Y, Yoshida H, Hirai M, Arakawa S, Yano I, Iwata K, Tokimatsu. Long-term efficacy of comprehensive multidisciplinary antibiotic stewardship programs centered on weekly prospective audit and feedback. Infection. 2018; doi:10.1007/s15010-017-1099-8.

[18] Barros LLS, Maia CSF, Monteiro MC. Fatores de risco associados ao agravamento de sepse em pacientes em Unidade de Terapia Intensiva. Cad. saúde colet. 2016; doi:10.1590/1414-462×201600040091.

\section{Supplementary Files}

This is a list of supplementary files associated with this preprint. Click to download.

- Additionalfile1.docx

- Additionalfile2.docx 